\title{
Cell proliferation activity in posterior uveal melanoma after Ru-106 brachytherapy: an EORTC ocular oncology group study
} Jacob Pe'er, Fritz H Stefani, Stefan Seregard, Tero Kivela, Peter Lommatzsch,
Jan U Prause, Beate Sobottka, Bertil Damato, Itay Chowers
Department of Ophthalmology, Hadassah University Hospital, Jerusalem, Israel

J Pe'er

I Chowers

Department of Ophthalmology, University Clinic, Munich, Germany F H Stefani

Ophthalmic Pathology and Oncology Service, St Erik's Eye Hospital, Stockholm, Sweden

S Seregard

Department of Ophthalmology, Helsinki University Central Hospital, Helsinki, Finland T Kivela

Department of Ophthalmology, University of Leipzig, Leipzig, Germany

P Lommatzsch

Eye Pathology Institute, University of Copenhagen, Copenhagen, Denmark J U Prause

Department of Ophthalmology, University of Tubingen, Tubingen, Germany

B Sobottka

St Paul's Eye Unit, Royal Liverpool University Hospital, Liverpool, UK B Damato

Correspondence to: Jacob Pe'er, MD, Department of Ophthalmology, Hadassah University Hospital, PO Box 12000, 91120 Jerusalem, Israel

peer@md2.huji.ac.il

Accepted for publication 4 April 2001
Abstract

Aim-To evaluate the cell proliferation activity in posterior uveal melanomas after Ru-106 brachytherapy.

Methods-Eyes containing choroidal or ciliary body melanoma from seven ocular oncology centres, which were enucleated after first being treated by Ru-106 brachytherapy and which had enough melanoma tissue to enable histological assessment, were included. The 57 eligible specimens were divided into a group of 44 eyes that were enucleated because of tumour regrowth, and a non-recurrent group of 13 eyes that were enucleated because of complications such as neovascular glaucoma. 46 non-irradiated eyes harbouring uveal melanoma served as a control group. All specimens underwent routine processing. They were cut into $5 \mu \mathrm{m}$ sections, and were stained with two main cell proliferation markers: PC-10 for PCNA and MIB-1 for Ki-67. The stained sections were assessed, and the cells that were positive in the immunostaining were counted in each section. The results were evaluated by various statistical methods.

Results-The PC-10 score showed a statistically significant difference across the three groups $(p=0.002)$. The control group showed the highest PC-10 score (median 31.0 PCC/HPF) followed by the tumour regrowth group (median 4.9 PCC/ HPF). The lowest PC-10 scores were found in the non-recurrent tumours (median 0.05 PCC/HPF). The MIB-1 score in the control group (median 5.77 PCC/HPF) was similar to the regrowth group (median 5.4 PCC/HPF). In contrast, the MIB-1 score in the non-recurrent tumours was statistically significantly lower (median 0.42 PCC/HPF). The PC-10 and MIB-1 scores were similar in tumours composed of either spindle cells or epithelioid cells in all groups.

Conclusions-The non-recurrent melanomas demonstrate significantly lower cellular proliferation activity than melanomas that showed regrowth or that were not irradiated at all. In our hands, PCNA gave more meaningful information than Ki-67. Our findings strongly support the need for treating regrowing posterior uveal melanoma either by enucleation or re-treatment by brachytherapy. On the other hand, also in the non-recurrent uveal melanomas there are viable cells with potential for proliferation, although fewer in number, with unknown capacity for metastatic spread. Therefore, the irradiated tumours should be followed for many years, probably for life.

(Br f Ophthalmol 2001;85:1208-1212)

The most common eye conserving way of treating posterior uveal melanoma is by radiotherapy. This is usually delivered by means of episcleral radioactive plaques. Cobalt- $60,{ }^{1}$ ruthenium-106, and iodine- $125^{3}$ are the radioactive materials most commonly used. Other methods of radiotherapy of posterior uveal melanoma include teletherapy using charged particles of proton ${ }^{4}$ and helium ion beams ${ }^{5}$; recently, stereotactic radiosurgery using the Leksell gamma knife has been described as being efficient. ${ }^{67}$

After radiotherapy, most uveal melanomas show significant reduction in size, although complete disappearance is not common. Histological evaluation of residual tumours have demonstrated that most of the tumours harbour viable melanoma cells, ${ }^{8-16}$ and some of these cells show proliferating activity using immunohistochemical markers. ${ }^{17-20}$

Previous studies have reported that between $6 \%$ to $34 \%$ of eyes treated for posterior uveal melanoma by radiotherapy are eventually enucleated, either because of regrowth of the tumour or because of complications caused by the radiotherapy, mainly secondary neovascular glaucoma..$^{7-21}$

The purpose of the present multicentre, multinational study was to evaluate the cell proliferation activity in posterior uveal melanoma after Ru-106 brachytherapy in eyes that were enucleated for various reasons. We have used two common cell proliferation markers, PC-10 for PCNA and MIB-1 for Ki-67. We also compared the findings in irradiated posterior uveal melanomas with those in tumours that were enucleated without being previously irradiated.

\section{Methods}

Eyes enucleated because of choroidal or ciliary body melanoma first treated with Ru-106 brachytherapy, and which had enough melanoma tissue to enable histological assessment, were included. Eyes that were enucleated after brachytherapy, but in which the primary method of treatment was unknown or in which histological sections did not contain 
Table 1 Clinical and histological features of the uveal melanomas in the study

\begin{tabular}{llll}
\hline & \multicolumn{2}{c}{ Brachytherapy } & \\
\cline { 2 - 3 } & Non-recurrent & \multicolumn{1}{c}{ Regrowth } & Control \\
\hline Location (choroid/ciliary body) & $11 / 2$ & $41 / 3$ & $42 / 5$ \\
Tumour height (mm)(SD) & $5.2(3)$ & $6(3)$ & $6.7(3.4)$ \\
Largest tumour dimension (mm)(SD) & $10(2.3)$ & $11.4(4.5)$ & $12.5(3.9)$ \\
Sex (F/M) & $5 / 8$ & $22 / 22$ & $19 / 28$ \\
Age (years)(SD) & $56(12)$ & $56(14)$ & $57(12)$ \\
Cell type (number of tumours): & 8 & 22 & 20 \\
$\quad$ Spindle & 3 & 20 & 26 \\
$\quad$ Epithelioid & 2 & 2 & - \\
$\quad$ Necrotic & & &
\end{tabular}

Tumour location, height and largest tumour diameter were determined according to the histological sections after enucleation.

enough tissue to enable reliable immunostaining, were excluded.

Tissue blocks from 143 cases of uveal melanoma were collected from the ophthalmic pathology laboratories of six European departments of ophthalmology. All eyes enucleated in these departments during the years 1980-97, after being previously treated with $\mathrm{Ru}-106$ plaque brachytherapy for uveal melanoma, were evaluated for the study. Of the 143 specimens collected, 57 were included in the study. The other 86 tumours were excluded because the paraffin block did not contain sufficient residual tumour. Two groups were formed according to the indication for enucleation: the first included 44 eyes that were enucleated because of tumour regrowth after the brachytherapy; the second group included the remaining 13 eyes that were enucleated following brachytherapy due to complications such as glaucoma or a blind and painful eye without evidence of tumour regrowth-the nonrecurrent group. Forty six non-irradiated consecutive uveal melanomas were obtained from the ophthalmic pathology laboratory of the Hadassah University Hospital for use as the control group.

Tumours from all laboratories had been fixed for at least $24-48$ hours in 10\% neutral buffered formalin before original tissue processing. Formalin fixed, paraffin embedded sections were stained with haematoxylin and eosin. Tumour location was categorised as follows: ciliary body melanoma was defined as a uveal melanoma of which more than two thirds of the tumour mass was located in the ciliary body; other tumours were defined as choroidal melanoma. Tumour cell typing was defined as epithelioid (containing epithelioid cells), or spindle (tumour without epithelioid cells). In order to evaluate similar areas of the tumour, serial sections of paraffin tissue blocks were cut at $5 \mu \mathrm{m}$ thickness, and immunostainings were performed on sections adjacent to that of the haematoxylin and eosin stain. Bleaching was used in sections from heavily pigmented tumours to enable immunostaining assessment.

Sections were deparaffinised in xylene and alcohols and placed for 15 minutes in 3\% alcoholic $\mathrm{H}_{2} \mathrm{O}_{2}$ to block endogenous peroxidase. In order to reveal masked antigen, slides stained with MIB-1 were placed in $10 \mathrm{mM}$ citrate buffer ( $\mathrm{pH}$ 6.0), treated in the microwave for 15 minutes, and then the container was removed from the microwave for cooling for 15 minutes. Slides stained with PC-10 were not treated in a microwave. Both MIB-1 and PC-10 stained slides were placed in phosphate buffered saline (PBS, $\mathrm{pH}$ 7.6). Sections were then treated with bovine serum albumin (BSA) to prevent background staining, and incubated for 1 hour with the primary antibody, either MIB-1 or PC-10 (Zymed Laboratories, Inc, San Francisco, CA, USA), at room temperature in a humidified chamber. Slides were rinsed in PBS for 3-4 minutes and incubated with biotin linked secondary antibody for 30 minutes and with the labelling reagent peroxidase conjugated streptavidin for 30 minutes (Bio Genex Laboratories, San Ramon, CA, USA). After rinsing, the peroxidase label was demonstrated using 3-amino-9-ethylcarbazole (AEC) for 15 minutes, and counterstained with Mayer's haematoxylin. AEC produces a red end product that is soluble in alcohol and is used with an aqueous mounting medium (Kaiser's glyceryl gelatin). A negative control was run using the same technique but omitting the primary antibody and adding the streptavidinbiotin complex.

The MIB-1 immunostaining was assessed by two observers who were masked to the light microscopic classification of the tumour as described by Seregard and his colleagues. ${ }^{19}$ In each section, 10 high power fields $(\times 40)$ in areas of maximal immunoreactivity were assessed; all melanoma cells that contained a distinct positive nuclear stain were regarded as positive. The mean MIB-1 and PC-10 positive cell count (PCC) for high power field (HPF) were calculated for each observer in each section.

In order to estimate the correlation between two quantitative parameters (such as tumour height and proliferative activity), the Spearman correlation coefficient was calculated, and its significance was assessed. When the mean levels of a quantitative parameter were compared in different categories of a qualitative parameter (such as different groups) with more than two categories, the ANOVA classic test was

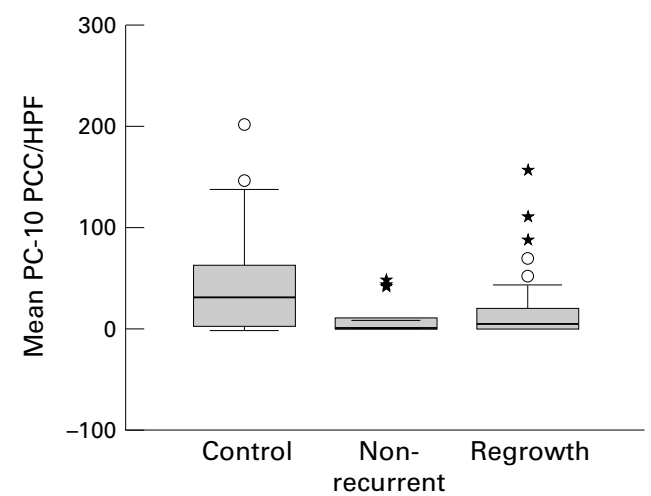

Figure 1 Box plot analyses of the proliferative activity of melanoma cells in the control, non-recurrent, and regrowth groups as estimated by PC-10 immunostaining. The thick horizontal lines show the median of the distribution of PC-10 positive cell count (PCC) per HPF, the boxes represent the interquartile range (IQR), and individual circles and asterisks represent outliers. A circle is more than 1.5 times $I Q R$ above the upper quartile, and an asterisk is more than three times IQR above the upper quartile. 


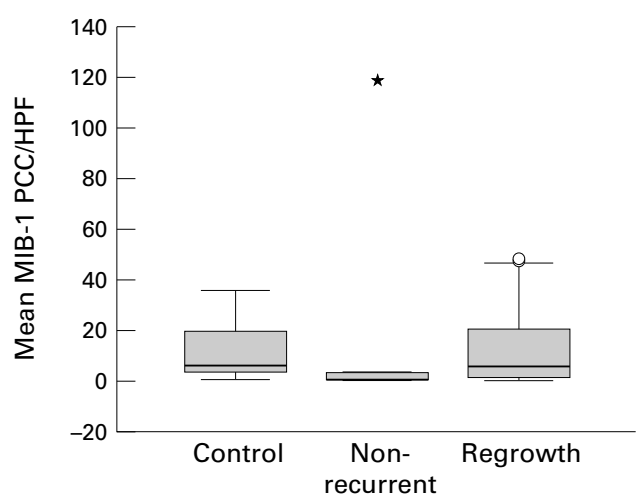

Figure 2 Box plot analyses of the proliferative activity of melanoma cells in the control, non-recurrent, and regrowth groups as estimated by MIB-1 immunostaining. The thick horizontal lines show the median of the distribution of MIB-1 positive cell count (PCC) per HPF, the boxes represent the interquartile range (IQR), and individual circles and asterisks represent outliers. A circle is more than 1.5 times IQR above the upper quartile, and an asterisk is more than three times $I Q R$ above the upper quartile.

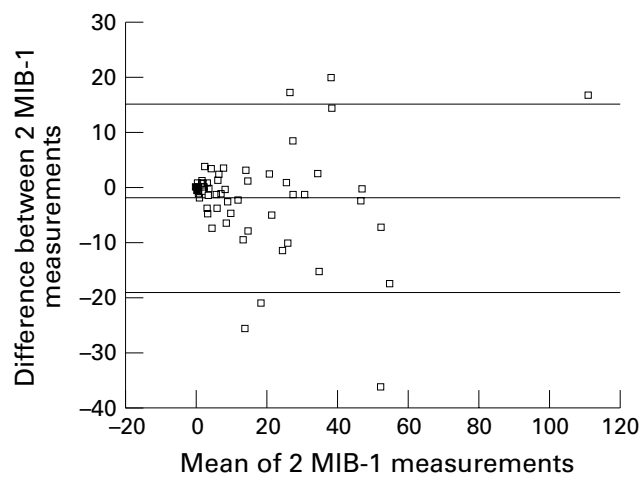

Figure 3 Interobserver agreement on the MIB-1 score

applied (including multiple pairwise comparison). The distribution of each quantitative parameter was estimated by using the Kolgmogorov-Smirnof test as well as the normal probability plot. When a parameter was not normally distributed the non-parametric Kruskal-Wallis ANOVA was used. MIB-1 and PC-10 readings were compared using the nonparametric Kruskal-Wallis ANOVA. If significance was found, multiple pairwise comparisons were performed using the Mann-Whitney non-parametric test with the Bonferroni correction for the significance level. The association between two qualitative parameters was tested using Fisher's exact test.

Results

Tumour location, height, and diameter, patient age, sex, and cell type of the tumours are

Table 2 The positive MIB-1 and PC-10 cell count per high power field according to the cell type

\begin{tabular}{lllll}
\hline \multirow{2}{*}{$\begin{array}{l}\text { Group/cell type } \\
\text { Regrowth }\end{array}$} & & Spindle & Epithelioid & \\
\cline { 3 - 4 } & & Median (range) & Median (range) & $\begin{array}{l}\text { Palue } \\
\text { (Mann-Whitney) }\end{array}$ \\
\hline \multirow{2}{*}{ Non-recurrent } & MIB-1 & $1.8(0-48.8)$ & $8.8(0-48.15)$ & 0.11 \\
& PC-10 & $5.7(0-88.4)$ & $4.92(0-157.6)$ & 0.55 \\
Control & MIB-1 & $0.7(0-2.6)$ & $0.15(0-119.8)$ & 1.0 \\
& PC-10 & $0.32(0-49.15)$ & $0(0-46.1)$ & 0.397 \\
Total (all groups) & MIB-1 & $5.82(0-35.65)$ & $6.05(0-35.35)$ & 0.942 \\
& PC-10 & $30(0-202.05)$ & $27.95(0-145.75)$ & 0.565 \\
& MIB-1 & $1.12(0-48.8)$ & $6.07(0-119.8)$ & 0.221 \\
& PC-10 & $4.35(0-88.4)$ & $1.97(0-157.6)$ & 0.195 \\
\hline
\end{tabular}

presented in Table 1. Except for cell type, there was no significant difference in these parameters across the three groups.

MIB-1 and PC-10 positive cells were identified in tumours from all groups. The mean positive PC-10 and MIB-1 cell count per high power field (PCC/HPF) are presented in Figures 1 and 2 respectively. The results of both PC-10 and MIB-1 assessment were not normally distributed and were therefore studied using non-parametric statistical tests. PC-10 score showed significant difference across the groups. Tumours treated by enucleation without previous brachytherapy showed the highest PC-10 score (median 31.0 PCC/HPF, range: 0-202 PCC/HPF), followed by tumours enucleated as a result of tumour regrowth after brachytherapy (median 4.9 $\mathrm{PCC} / \mathrm{HPF}$, range 0-157.6 PCC/HPF). The lowest PC-10 score was found in the nonrecurrent tumours from eyes enucleated because of complications other than tumour regrowth (median $0.05 \mathrm{PCC} / \mathrm{HPF}$, range $0-49.1 \mathrm{PCC} / \mathrm{HPF})$. These differences in the PC-10 score were significant $(p=0.002$, Kruskal-Wallis test).

The MIB-1 score of tumours enucleated without previous brachytherapy (median 5.8 $\mathrm{PCC} / \mathrm{HPF}$, range $0-35.65 \mathrm{PCC} / \mathrm{HPF}$ ) was similar to the MIB-1 score of tumours enucleated because of tumour regrowth after Ru-106 brachytherapy (median 5.4 PCC/HPF, range 0-48.8 PCC/HPF). By contrast, the MIB-1 score of the non-recurrent tumours was smaller (median $0.42 \mathrm{PCC} / \mathrm{HPF}$, range 0-119.8 PCC/HPF). This difference in the MIB-1 score across the groups, although significant in pairwise comparisons, was not significant after the Bonferroni correction for the significance level ( $p=0.065$, KruskalWallis test). There was a good interobserver agreement on the MIB-1 PCC/HPF between the two observers (Fig 3).

Table 2 shows the MIB- 1 and PC-10 scores of each group according to the cell type. The PC-10 and MIB-1 scores were similar in tumours composed of either spindle or epithelioid cell type in all groups.

Of the PCNA stained tumours, stained cells were not shown in $8 / 46$ tumours $(17.4 \%)$ in the control group, in $11 / 44$ tumours (25\%) in the regrowth group, and in 6/13 tumours $(46.2 \%)$ in the non-recurrent tumours. Of the Ki-67 stained tumours, no stained cells were found in $6 / 46$ tumours $(13.0 \%)$ of the control group, in $5 / 44$ tumours $(11.4 \%)$ of the regrowth group, and in 3/13 tumours $(23.1 \%)$ in the non-recurrent group.

\section{Discussion}

The diagnosis of uveal melanoma as well as the follow up of these tumours after conservative, usually irradiation, treatment, is done by non-invasive methods, mainly funduscopy and B-scan and A-scan ultrasonography. As opposed to most malignancies, in uveal melanoma we often do not have tissue diagnosis, and we do not have histological follow up of the residue of the tumour. The reason for this is the fear of spreading tumour cells beyond the 
eye, through the site of a biopsy entrance or through tumour vessels damaged by the needle. By fundus examination we can see the reaction of the posterior uveal melanoma to irradiation. Ultrasound examination, mainly the A-scan, guides us in evaluating the size of the tumour (mainly the height) and the internal reflectivity, which indicate tissue characteristics of the tumour. We usually view shrinkage in tumour size and increase of the internal reflectivity as indicators of success of the irradiation treatment. ${ }^{22}$

It is well known that most posterior melanomas do not disappear completely after irradiation treatment, but show significant reduction in size. The remnants of the tumours must be followed to detect regrowth indicating activation. Reduction in the tumour size and partial flattening have been shown to stabilise after about 2 years. However, as long as tumour remnants remain the tumour should be followed for possible regrowth.

All histological studies of uveal melanoma remnants show viable tumour cells, mainly of the spindle cell type. ${ }^{8-16}$ Moreover, cell proliferation has been found in several studies of irradiated tumours. ${ }^{17-20}$

Various methods have been used to assess cellular proliferation in uveal melanoma. Among them are counting of mitoses, measurement of DNA content by thymidine labelling, incorporation of thymidine analogue such as bromodeoxyuridine, ${ }^{23}$ flow cytometry, ${ }^{24}$ and assessment of silver stained nucleolar organiser regions. In recent years immunohistochemical markers, such as $\mathrm{PCNA}^{17{ }^{19}}$ and $\mathrm{Ki}-67,{ }^{18}{ }^{20}$ have been used to examine cell proliferation in conventionally processed histological preparations. The markers seem to be reliable and easy tools for evaluating cellular activity.

In the present study we have used the two main proliferation markers, PC-10 for PCNA and MIB-1 for Ki-67, to evaluate the cell proliferation activity in posterior uveal melanomas after being treated by Ru-106 brachytherapy. We have studied two distinct groups of residual tumours: those which showed regrowth and actually failure of the treatment, and those that were enucleated because of post-irradiation complications. Because well controlled tumours did not always yield adequate tissue for study, our sample is overrepresented by regrowing tumours. We compared these groups with a control group of non-irradiated melanomas of similar sizes.

Both immunostainings demonstrated that the non-recurrent tumours had significantly lower cellular proliferation activity than the control group and the regrowth group. The regrowth group showed significantly less proliferative activity than the control group by PC-10 staining for PCNA. However, we could not show a difference between the control group and the regrowth group when we used the Ki-67 immunostaining. The reason for this may be manifold. Firstly, the Ki-67 and PCNA proteins are distinctly different cell cycle associated antigens. Secondly, the long half life of
PCNA will in most cases generate a larger proportion of immunopositive cells than Ki-67. Compared with flow cytometry, PC-10 tends to overestimate and MIB-1 to underestimate cell proliferation. PC-10 will stain cells even after they have left the cell cycle, because of long half life. In this sense it is more "sensitive" to cells that are cycling or recently left the cell cycle. This also explains why more cells stain with PC-10 than with MIB-1.

This study is the first to compare the usefulness of these markers in uveal melanoma after failed brachytherapy. PC-10 was the only immunostaining to detect a difference between regrowing tumours and controls, owing to its higher sensitivity, and therefore in our hands it provides more meaningful information. Our results are comparable with previous studies that examined cell proliferation activities by one of the markers we used in our study. ${ }^{17-20}$ The measurements by both markers supplement each other and give a fuller picture than does either alone.

Counting the number of tumours in which proliferating tumour cells were not detected, we found significantly more tumours with zero proliferating cells in the non-recurrent group. Also in this counting, the results with PCNA marker proved to be more meaningful. These results are also comparable with previous studies. ${ }^{17} 19$

It is obvious that clinically detectable regrowth of tumours indicate activity of the tumour, and our results are not surprising. Without having histological markers, ocular oncologists tend to enucleate or to re-treat regrowing uveal melanomas. Our findings strongly support this attitude, since regrowing tumours may have a compartment of cycling cells as large as non-irradiated tumours, and since the presence of such proliferating cell population was previously shown to be associated with death from metastatic disease. ${ }^{25}$

On the other hand, we cannot ignore the apparent proliferating activity, although low, of part of the non-recurrent tumours that were enucleated because of ocular complications, the group that comprises the tumours that reacted well to irradiation. Apparently, these viable tumours remain a potential for later regrowth, and should be followed carefully for many years and probably for life. It remains unclear if these tumours, successfully treated by clinical standards, maintain a capacity for metastatic spread.

\footnotetext{
1 Stallard, HB. Malignant melanoma of the choroid treated with radioactive applicators. Trans Ophthalmol Soc UK 1959;79:373-92.

2 Lommatzsch PK. Experiences in the treatment of malignant melanoma of the choroid with ${ }^{106} \mathrm{Ru} /{ }^{106} \mathrm{Rh}$ beta-ray applicators. Trans Ophthalmol Soc UK 1973;93:119-32.

3 Sealy R, le Roux PLM, Rapley F, et al. The treatment of ophthalmic tumors with low-energy sources. $\mathrm{Br} \mathcal{F}$ Radiol 1976;49:551-4.

4 Gragoudas ES, Goitein M, Verhey L, et al. Proton beam rradiation: an alternative to enucleation for intraocular melanomas. Ophthalmology 1980;87:571-81.

5 Char DH, Saunders W, Castro JR, et al. Helium ion therapy for choroidal melanoma. Ophthalmology 1983;90:1219-25.

6 Zehetmayer M, Kitz K, Menapace R, et al. Local tumor control and morbidity after one to three fractions of stereotactic external beam irradiation for uveal melanoma. Radiother Oncol 2000;55:135-44.
} 
7 Mueller AJ, Talies S, Schaller UC, et al. Stereotactic radiosurgery of large uveal melanomas with the gamma-

8 MacFaul PA, Morgan G. Histopathological changes in malignant melanomas of the choroid after cobalt plaque therapy. Br f Ophthalmol 1977;61:221-8.

9 Lommatzsch PK. Results after $\beta$-irradiation $\left({ }^{106} \mathrm{Ru} /{ }^{106} \mathrm{Rh}\right)$ of choroidal melanomas: 20 years' experience. $\mathrm{Br} \mathcal{F}$ Ophthalmol 1986;70:844-51.

10 Crawford, JB, Char DH. Histopathology of uveal melanomas treated with charged particle radiation. Ophthalmology 1987;94:639-43

11 Shields CL, Shields JA, Karlsson U, et al. Enucleation after plaque radiotherapy for posterior uveal melanoma. Histopathologic findings. Ophthalmology 1990:97:1665-70.

12 Saornil MA, Egan KM, Gragoudas ES, et al. Histopathology of proton beam-irradiated vs. enucleated uveal ogy of proton beam-irradiated vs. enucleat
melanomas. Arch Ophthalmol 1992;110:1112-18.

13 Messmer E, Bornfeld N, Foerster M, et al. Histopathologic findings in eyes treated with a ruthenium plaque for uveal findings in eyes treated with a ruthenium plaque for uveal 391-6.

14 Schilling H, Bornfeld N, Friedrichs W, et al. Histopathologic findings in large uveal melanomas after brachytherapy with iodine 125 ophthalmic plaque. Ger $\mathcal{F}$ Ophthalmo 1994;3:232-8

15 Petrovich Z, McDonnell JM, Palmer D, et al. Histopathologic changes following irradiation for uveal tract melanoma. Am f Clin Oncol 1994;17:298-306.

16 Saornil MA, Fisher MR, Campbell RJ, et al. Histopathologic study of eyes after iodine 125 episcleral plaque irradiation for uveal melanoma. Arch Ophthalmol 1997;115:1395-400.
17 Pe'er J, Gnessin H, Shargal Y, et al. PC-10 immunostaining of proliferating cell nuclear antigen in posterior uveal melanoma. Ophthalmology 1994;101:56-62.

18 Schilling H, Sehu KW, Lee WR. A histologic study (including DNMA quantification and $\mathrm{Ki}-67$ labeling index) in uveal melanomas after brachytherapy with ruthenium plaques. Invest Ophthalmol Vis Sci 1997;38:2081-92.

19 Seregard S, Lundell G, Lax I, et al. Tumor cell proliferation after failed ruthenium plaque radiotherapy for posterior uveal melanoma. Acta Ophthalmol Scand 1997;75:148-54.

20 Chiquet C, Grange JD, Ayzac L, et al. Effects of proton beam irradiation on uveal melanomas: a comparative study of $\mathrm{Ki}-67$ expression in irradiated versus non-irradiated melanomas. Br $\mathcal{F}$ Ophthalmol 2000;84:98-102.

21 Lommatzsch PK, Werschnik C, Schuster E. Long-term follow-up of $\mathrm{Ru}-106 / \mathrm{Rh}-106$ brachytherapy for posterior uveal melanoma. Graefes Arch Clin Exp Ophthalmol 2000;238:129-37.

22 Ossoinig KC. Standardized echography: basic principles, clinical applications, and results. Int Ophthalmol Clin 1979; 19:127-210

23 Char DH, Crawford JB, Kaleta-Michaels S, et al. Analysis of radiation failure after uveal melanoma brachytherapy. $A m \mathcal{F}$ Ophthalmol 1989;108:712-16.

24 Shapiro BE, Felberg NT, Donoso LA, et al. Flow cytometry of uveal melanomas. Cancer Biochem Biophys 1986;8:2358.

25 Seregard S, Oskarsson M, Spangberg B. PC-10 as a predictor of prognosis after antigen retrieval in posterior uveal melanoma. Invest Ophthalmol Vis Sci 1996;38:1451-8. 